LA GRANJA:

REVISTA DE

CIENCIAS DE LA VIDA

pISSN:1390-3799; eISSN:1390-8596

http://doi.org/10.17163/lgr.n25.2017.06
Artículo científico / Scientific paper

Ciencias Veterinarias

\title{
DETERMINACIÓN DE LOS VALORES DE REFERENCIA EN EL HEMOGRAMA DE CABALLOS NACIDOS O CRIADOS A MÁS DE 3000 M.S.N.M. EN LA SIERRA CENTRO NORTE ECUATORIANA
}

DETERMINATION OF THE REFERENCE VALUES IN THE BLOOD COUNT OF

HORSES BORN OR REARED MORE THAN 3000 M.A.S.L. IN THE NORTH-

CENTRAL HIGHLANDS OF ECUADOR

\section{José Luis Izurieta Barzola, Diego Fernando Luna Narváez*, Yolanda Mercedes Cedeño Prócel y Sergio Rolando Chacha Vega}

Universidad Central del Ecuador, Jerónimo Leiton s/n y Gatto Sobral, Teléfono 593(02) 256 6160, Quito, Ecuador.

*Autor para correspondencia: dluna@doctor.com

Manuscrito recibido el 16 de junio de 2016. Revisado el 19 de julio de 2016. Aceptado el 19 de diciembre de 2016. Publicado el 30 de diciembre de 2016.

\begin{abstract}
Resumen
En la sierra centro norte ecuatoriana se realizó un estudio de hemograma a 100 caballos mayores de dos años de edad, criados sobre los 3000 m.s.n.m., el objetivo de esta investigación fue determinar valores hematológicos a esta altitud y compararlos con otros estudios realizados a nivel del mar. Las muestras sanguíneas fueron tomadas en reposo de los animales y enviadas al laboratorio para ser analizadas en el equipo de hematología automática Mindray $(B \mathrm{BC} 2800$ Vet, para el análisis de los datos y determinar los valores de referencia se utilizó el complemento de Microsoft Excel (RReference Value Advisor. Las muestras fueron tomadas de caballos criollos ecuatorianos para obtener datos hematológicos de este tipo de caballos antes no estudiado. Se encontraron 82 muestras idóneas para establecer valores de hemograma con distribución normal, glóbulos rojos $6,23-10,84 \times 10^{6} / \mathrm{mL}$; hemoglobina $11,4-18,4 \mathrm{~g} / \mathrm{L}$; hematocrito 32,3 - 52,3\%; volumen corpuscular medio 40,2 - 57 fL; hemoglobina corpuscular media $14-20,3 \mathrm{pg}$; concentración de hemoglobina corpuscular media $32-37 \mathrm{~g} / \mathrm{L}$; glóbulos blancos $4,8-12 \times 10^{3} / \mathrm{mL}$; linfocitos $1,4-7,5 \times 10^{3} / \mathrm{mL}$; monocitos $0,2-0,7 \times 10^{3} / \mathrm{mL}$; granulocitos $2,2-8,8 \times 10^{3} / \mathrm{mL}$; linfocitos $\% 16,8-64,9 \%$, monocitos \% 2,9-7\%, granulocitos \% 30,5-78,7\%; plaquetas $101-401 \times 10^{3} / \mathrm{mL}$. Los resultados se compararon con referencias bibliográficas internacionales y se encontró diferencias significativas $(\mathrm{P}<0,05)$ en glóbulos rojos, hemoglobina, hematocrito y monocitos en el estudio de Böhmwald et al., (1984); diferencia significativa en hematocrito, volumen corpuscular medio, linfocitos, monocitos y plaquetas con el estudio de Giordano et al., (2008); mientras que con el estudio de Díaz et al., (2011) hubo diferencia significativa con la hemoglobina, monocitos y plaquetas.
\end{abstract}

Palabras claves: caballos, eritropoyetina, hemoglobina, hemograma, hipoxia. 


\begin{abstract}
In the north central Ecuadorian highland, a blood count was performed on 100 horses over two years of age, raised over 3000 m.a.s.l., the objective of this investigation was to determine hematological values at this altitude and compare them with other studies performed at sea level. The blood samples were taken at rest from the animals and sent to the laboratory to be analyzed in the automatic hematology equipment Mindray $(B B C 2800$ Vet, for the analysis of the data and to determine the reference values we used the Microsoft Excel add-on $®$ Reference Value Advisor. Samples were taken from Ecuadorian Creole horses to obtain hematological data of this type of horses previously untested. Was found 82 samples suitable for establishing blood count values with normal distribution, red blood cells $6,23-10,84 \times 10^{6} / \mathrm{mL}$; hemoglobin $11,4-18,4 \mathrm{~g} / \mathrm{L}$; hematocrit $32,3-52,3 \%$, mean corpuscular volume 40,2 - $57 \mathrm{fL}$; Mean corpuscular hemoglobin $14-20,3$ pg; Mean corpuscular hemoglobin concentration $32-37 \mathrm{~g} / \mathrm{L}$; white blood cells $4,8-12 \times 10^{3} / \mathrm{mL}$; lymphocytes $1,4-7,5 \times 10^{3} / \mathrm{mL}$; monocytes $0,2-0,7 \times 10^{3} / \mathrm{mL}$; granulocytes $2,2-8,8 \times 10^{3} / \mathrm{mL}$; lymphocytes $\% 16,8-64,9 \%$, monocytes $\% 2,9-7 \%$, granulocytes $\% 30,5-78,7 \%$; platelets $101-401 \times 10^{3} / \mathrm{mL}$. The results were compared with international bibliographical references and significant differences $(\mathrm{P}<0,05)$ in red blood cells, hemoglobin, hematocrit and monocytes were found in the study of Böhmwald et al., (1984), a significant difference in hematocrit, mean corpuscular volume, lymphocytes, monocytes and platelets with the study of Giordano $e t$ al., (2008); While with the study of Díaz et al., (2011) significant difference with hemoglobin, monocytes and platelets.
\end{abstract}

Keywords: erythropoietin, hemoglobin, hemogram, horses, hypoxia.

Forma sugerida de citar: Izurieta Barzola, José Luis, Diego Fernando Luna Narváez, Yolanda Mercedes Cedeño Prócel y Sergio Rolando Chacha Vega. 2017. Determinación de los valores de referencia en el hemograma de caballos nacidos o criados a más de 3000 m.s.n.m. en la sierra centro norte ecuatoriana. La Granja: Revista de Ciencias de la Vida. Vol. 25(1):62-70. pISSN:1390-3799; eISSN:1390-8596. 


\section{Introducción}

La medicina de altura tiene gran importancia en medicina humana, al ascender a elevaciones cada vez mayores se ha hecho más importante comprender los efectos de la altura y de las bajas presiones de los gases sobre el cuerpo humano (Hall, 2016), lastimosamente éstos efectos no han tenido la misma relevancia en medicina veterinaria.

El ambiente de altura es considerado como un complejo ecológico multifactorial cuyo fenómeno natural determinante es la disminución de la presión atmosférica, a medida que se asciende se produce una disminución de la presión del oxígeno en el aire a respirar. A éste efecto físico directo se añaden otros factores como la sequedad del aire, el frío, los cambios en la calidad, variedad y disponibilidad de las pasturas, y las costumbres, los cuales de una $\mathrm{u}$ otra manera, intervienen en la aclimatación y las molestias o síntomas de un estado de malestar (Barranco et al., 2002).

Cuando se respira aire a nivel del mar la presión atmosférica es de $760 \mathrm{mmHg}$, este valor cambia con las condiciones meteorológicas y cae al incrementarse la altitud, al igual que la presión parcial de sus componentes (oxigeno, nitrógeno, dióxido de carbono, etc.) de la atmósfera. Así a los 3000 m.s.n.m. la presión atmosférica es de $526 \mathrm{mmHg}$ y la presión para el oxígeno 110,4 $\mathrm{mmHg}$. El oxígeno representa el $21 \%$ de los componentes de la atmósfera. Por encima de los 3000 m.s.n.m. los niveles de salud, productividad y supervivencia están en sus límites por la menor presión parcial de oxígeno (Barranco et al., 2002).

Para adaptarse a este ambiente, el organismo desarrolla ciertos cambios especialmente en los sistemas cardiovascular, respiratorio y hematopoyético. Los principales mecanismos mediante los cuales se produce la adaptación son: aumento de la ventilación pulmonar, aumento en el número de eritrocitos, aumento de la capacidad de difusión pulmonar, aumento de la vascularización de los tejidos periféricos, y aumento de la capacidad de las células tisulares para utilizar el oxígeno a pesar de una presión de oxigeno baja (Hall, 2016). Esto se traduce en una mayor capacidad del organismo por captar el aire y oxígeno atmosférico para utilizarlo de una forma más eficiente por los distintos órganos y tejidos, y así poder seguir cumpliendo con las exigencias que conlleva la actividad diaria en este ambiente de altura.
Por otro lado, los caballos son considerados los deportistas elites dentro de los animales, están dotados de una gran capacidad pulmonar, vascularización y abundante musculatura que junto a una conformación anatómica muy especializada les permiten galopar hasta alcanzar altas velocidades y mantenerla por largas distancias, el mejor ejemplo de esto es la famosa "Triple Corona", el máximo premio otorgado a los caballos pura sangre de carreras (Spc) en la cual un mismo caballo de 3 años de edad compite y gana las tres carreras de 1900, 2000 y 2400 metros, poniendo en manifiesto que poseen velocidad y resistencia incomparables.

McGorum et al. (2006) mencionan que la gran altitud puede tener un efecto alveolar incapacitante en la difusión de $\mathrm{O}_{2}$ alveolar, que puede ser importante en caballos de competencia. A una altitud de 3048 m.s.n.m. la presión atmosférica es de 522 $\mathrm{mmHg}(69.6 \mathrm{kPa})$, la cual es inadecuada para transferir oxígeno a los capilares pulmonares (Greene et al., 1999). Los caballos participan en varias disciplinas deportivas, tanto de agilidad, velocidad, precisión y en el campo aún se los utiliza como herramienta de trabajo, medio de transporte y sin duda como un fiel animal de compañía.

Al ser Latinoamérica y Ecuador lugares con un gran contraste geográfico, donde una importante parte de la población habita por sobre los 3000 m.s.n.m. surge la necesidad de investigar y conocer cómo se ven alterados los valores hematológicos de los caballos como punto de partida para forjar una medicina veterinaria de altura.

El hemograma es un examen muy utilizado en la clínica equina, pues es un indicador de alteraciones que no son siempre percibidas durante el examen clínico (Kazuko et al., 2009). Un hemograma completo podría servir como método diagnóstico para una enfermedad concreta, pero la mayoría de las veces se utiliza para conocer la condición general del individuo o su respuesta frente a la enfermedad (Cuenca y Pastor, 2006).

Los objetivos de este trabajo fueron establecer los parámetros hematológicos en caballos a más de 3000 metros sobre el nivel del mar y compararlos con los valores de otros autores a nivel del mar.

El habitante de altura posee un grado de eritrocitosis en respuesta a la hipoxia como un mecanismo de compensación, en equinos este porcentaje es desconocido por falta de estudios previos a dichas alturas.

Los mecanismos compensadores del sistema 
cardiovascular al enfrentarse a desafíos como el ejercicio, inflamación, cicatrización de heridas, cambios de altura nos llevan a querer conocer las modificaciones que se dan en el hemograma para crear un comportamiento equitativo al normal, como es el caso de los caballos en los cuales una descompensación se observa tardíamente, es decir cuando se han causado severas alteraciones en su fisiología.

Comprender mejor como es el cambio en su hematología, fisiología, y su capacidad para adaptarse a realizar todos los esfuerzos a los que son sometidos normalmente los caballos a alturas superiores a los 3000 m.s.n.m., nos permitirá realizar un mejor diagnóstico, tratamiento y mejorar el performance en su desempeño deportivo, por lo que se decidió realizar el estudio en zonas de la sierra centro norte ecuatoriana, debido a que las características de clima y ambiente son similares en estas zonas.

\section{Materiales y métodos}

El estudio se realizó en la sierra centro norte ecuatoriana, en seis zonas entre 3200 y 3800 metros sobre el nivel del mar. La mayoría fueron pequeñas fincas donde tenían los caballos al pastoreo, y otras comunidades donde los utilizan como medio de transporte.

\subsection{Factores de estudio}

En el presente estudio la altura fue superior a 3000 m.s.n.m., se analizó para determinar valores de referencia en el hemograma y si se alteraban los valores normales en caballos criados a esta altura.

El estudio consistió en tomar muestras de sangre venosa de la yugular externa de caballos clínicamente sanos para posteriormente ser procesadas en el laboratorio. Se realizaron hemogramas de todas las muestras con el equipo Mindray $(\mathrm{B} B C-2800 \mathrm{Vet}$ y se determinaron los valores de referencia de cada parámetro hematológico.

Para el segundo objetivo específico de la presente investigación se compararon los resultados de los valores del hemograma de caballos a más de 3000 m.s.n.m. frente a valores de referencia internacionales de otros autores a través de un análisis estadístico.

Los caballos utilizados para tomar las muestras sanguíneas fueron seleccionados previo los siguientes criterios de inclusión: caballos que nacieron o ha- yan sido criados a más de 3000 m.s.n.m., machos y hembras mayores a dos años de edad, en buen estado de salud, que no presentaron ninguna anormalidad en el examen clínico, de cualquier disciplina, se prefirió caballos criollos ecuatorianos para evitar variaciones en sus parámetros de composición sanguínea.

\subsection{Análisis estadístico}

Para realizar el análisis estadístico, se utilizó el programa Microsoft Excel (B)con el complemento Reference Value Advisor, el cual permitió determinar los límites de referencia de la muestra y eliminar valores anómalos llamados outliers a través del análisis de Dixon-Reed y Test de Tukey. El método utilizado fue un paramétrico con un nivel de confianza del $90 \%$ (Geffré et al., 2011). Se comprobó que la distribución de la muestra fue normal de acuerdo al test de normalidad de Anderson-Darling, con histogramas y Q-Q Plots. Se extrajeron las medidas de tendencia central: media, mediana, desviación estándar, valor máximo y mínimo y gráficos de distribución.

Para comparar los resultados encontrados con valores de referencia bibliográficas de otros autores, se optó por el método estadístico de prueba de hipótesis, Prueba $Z$ (diferencia significativa $>1,96$ ) para medias de dos muestras, por tratarse de una muestra mayor a 30 individuos y con distribución normal.

\subsection{Métodos específicos de manejo del ex- perimento}

Para la toma de la muestra, los caballos previamente permanecieron 20 minutos sujetados en un lugar tranquilo, para que no presenten alteraciones en su composición sanguínea por motivos de actividad física o estrés.

\subsubsection{Universo y marco del muestreo}

Es importante hacer hincapié en el hecho que no se dispone de un censo real de la población de caballos en el Ecuador, lo cual dificulta los estudios para esta especie, especialmente en estudios enfermedades infecciosas. Para establecer el marco del muestreo se utilizaron las directrices de La Asociación Americana de Patólogos Clínicos Veterinarios (ASVCP), donde indica que se puede utilizar un tamaño de 
muestra de $40 \leq x<120$ con un método paramétrico y nivel de confianza del $90 \%$ (Friedrichs et al., 2012).

\section{Resultados}

De las 100 muestras tomadas y procesadas, el programa Reference Value Advisor reportó 18 muestras como potenciales valores anómalos por lo que se eliminaron estas muestras para el estudio. La muestra total de datos utilizados para delimitar los valores de referencia del estudio finalmente fue de 82 (ver Tabla 1 y Figura 2).

Las edades de los caballos en estudio presentaron un rango mínimo y máximo de 2 y 20 años, con una media de 8,1 años y una desviación estándar de \pm 4.5 (ver Figura 1).

Tabla 1. Valores hematológicos de caballos nacidos o criados a más de los 3000 m.s.n.m. en la sierra centro norte ecuatoriana.

\begin{tabular}{lcccccc}
\hline Elemento & Valor & Media & Mediana & SD & Mínimo & Máximo \\
\hline Eritrocitos & $\times 10^{6} / \mathrm{mL}$ & 8,3 & 8,3 & 1 & 6,23 & 10,84 \\
Hemoglobina & $\mathrm{g} / \mathrm{dL}$ & 14,1 & 14,0 & 1,6 & 11,4 & 18,4 \\
Hematocrito & $\%$ & 41 & 41,0 & 4,4 & 32,3 & 52,2 \\
VCM & $\mathrm{fL}$ & 49,6 & 49,5 & 3,4 & 40,2 & 57 \\
$\mathrm{HCM}$ & $\mathrm{pg}$ & 16,9 & 17,1 & 1,1 & 14 & 20,3 \\
$\mathrm{CHCM}$ & $\mathrm{g} / \mathrm{dL}$ & 34,3 & 34,2 & 1 & 32 & 37 \\
Leucocitos & $10^{3} / \mathrm{mL}$ & 8,3 & 8,3 & 1,7 & 4,8 & 12 \\
Lym\# & $10^{3} / \mathrm{mL}$ & 3,6 & 3,6 & 1,2 & 1,4 & 7,5 \\
Mon\# & $10^{3} / \mathrm{mL}$ & 0,4 & 0,4 & 0,1 & 0,2 & 0,7 \\
Gran\# & $10^{3} / \mathrm{mL}$ & 4,3 & 4 & 1,4 & 2,2 & 8,8 \\
Plaquetas & $10^{3} / \mathrm{mL}$ & 239 & 225,5 & 68,2 & 101 & 401 \\
\hline SD:Desva
\end{tabular}

SD: Desviación estándar, VCM: Volumen corpuscular medio, HCM: Hemoglobina corpuscular media, CHCM: Concentración de hemoglobina corpuscular media.

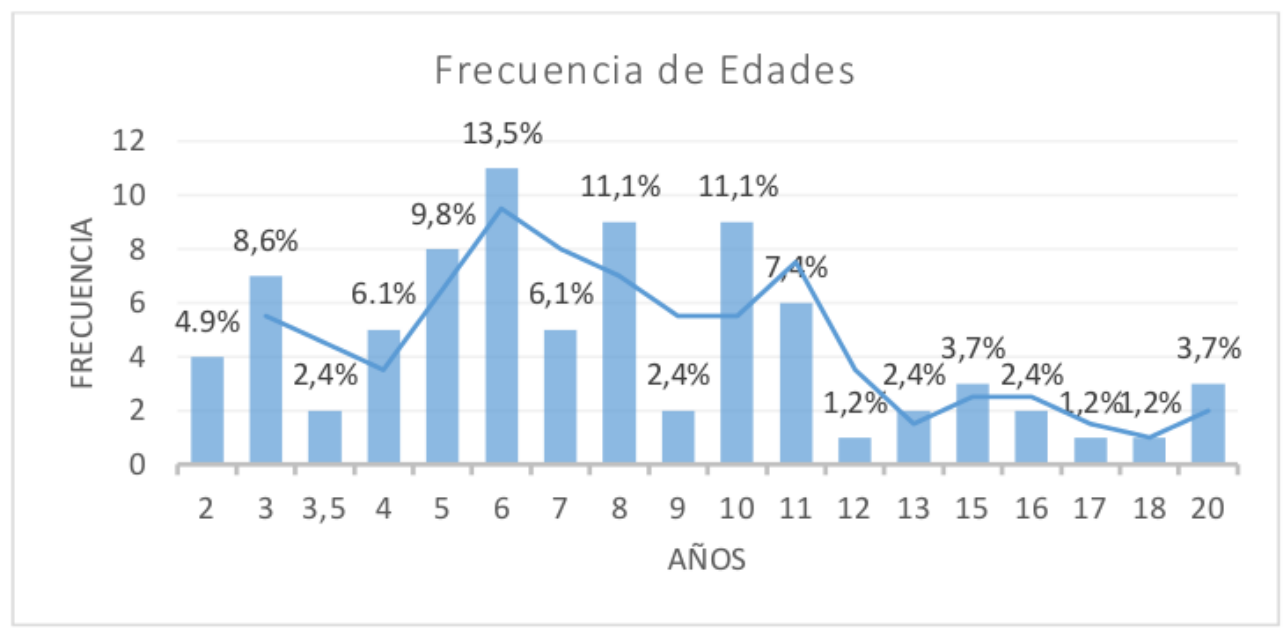

Figura 1. Frecuencia de edades de los caballos estudiados para la referencia hematológica. 
Eritrocitos

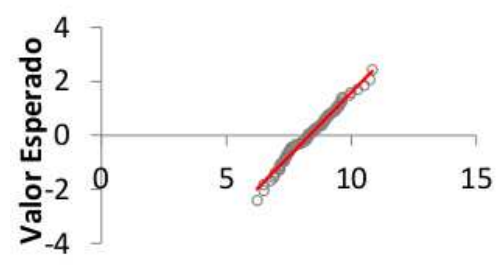

9,25

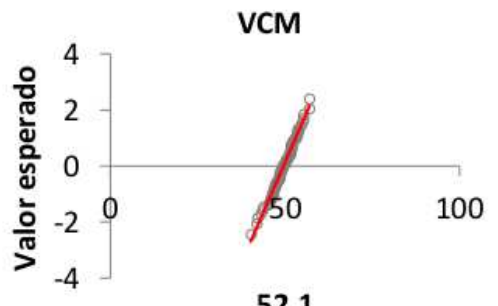

52,1

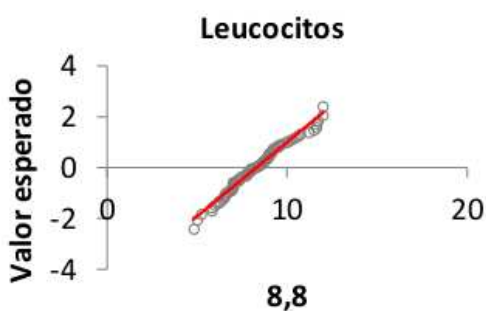

8,8

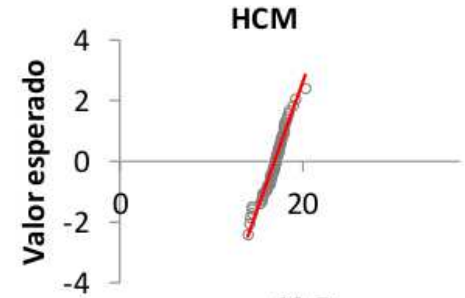

19,5

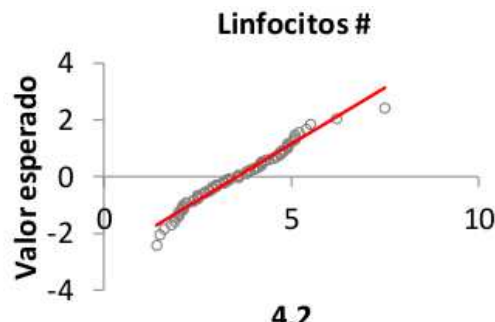

4,2

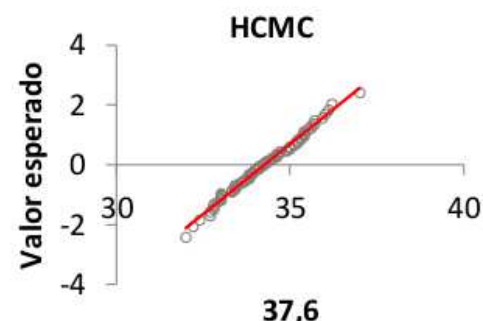

$$
37,6
$$

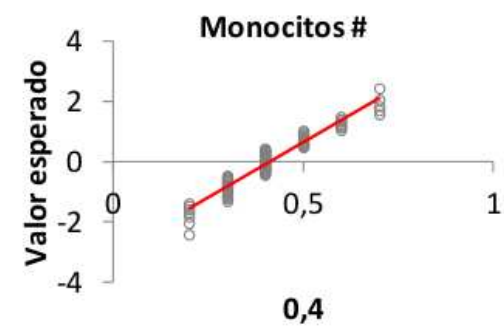

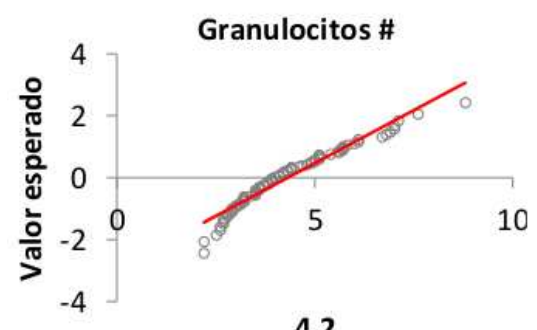

4,2

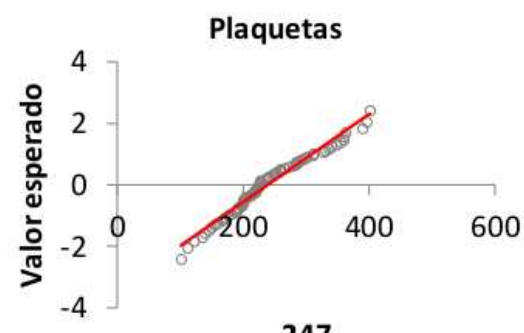

247

Figura 2. QQ- Plots de distribución de los elementos sanguíneos analizados por Reference Value Advisor. 
Tabla 2. Valores referenciales y prueba $\mathrm{Z}$ del hemograma de caballos a más de 3000 m.s.n.m. frente a referencias internacionales: Giordano et al., (2008), Böhmwald et al., (1984) y Díaz et al., (2011).

\begin{tabular}{|c|c|c|c|c|c|c|c|c|c|c|c|c|c|c|c|c|c|c|c|}
\hline & \multirow{2}{*}{ Elementos } & \multicolumn{4}{|c|}{ Caballos más de 3000 m.s n.m. } & \multicolumn{4}{|c|}{ Giordano et al., (2008), 120 m.s.n.m. } & \multicolumn{5}{|c|}{ Böhmwald et al., (1984), 14 m.s.n.m } & \multicolumn{5}{|c|}{ Díaz et al., (2011), 0-300 m.s.n.m. } \\
\hline & & Media & Min-Max & DS & Media & Min-Max & DS & Prueba Z & Probabilidad & Media & Min-Max & DS & Prueba Z & Probabilidad & Media & Min-Max & DS & Prueba Z & Probabilidad \\
\hline & Eritrocitos & 8,3 & $6,23-10,84$ & 1 & 8,62 & $4,01-11-29$ & 1,24 & -1.83 & 0,0336 & 6,98 & - & 0,8 & 5,48 & 1 & 8,3 & $6,5-10,4$ & 0,9 & 0 & 0,4999 \\
\hline & $\mathrm{HB}$ & 14,1 & $11,4-18,4$ & 1,6 & 14,48 & $10,6-18,94$ & 1,92 & $-1,25$ & 0,1056 & 12,32 & - & 1,3 & 4,56 & 1 & 13,9 & $11,4-17,1$ & 1,5 & 2,59 & 0,9952 \\
\hline & $\mathrm{HCT}$ & 41 & $32,3-52,2$ & 4,4 & 37,45 & $33,88-48,9$ & 4,97 & 5,27 & 1 & 35,82 & - & 3,78 & 4,62 & 1 & 40,7 & $33,3-52$ & 4,6 & 0,367 & 0,6405 \\
\hline 5 & $\mathrm{VCM}$ & 49,6 & $40,2-57$ & 3,4 & 43,65 & $38,35-49,44$ & 3,02 & 21,03 & 1 & 53,31 & - & 4,46 & $-1,72$ & 0,0427 & 48,4 & $20,7-57,8$ & 5,5 & 1,37 & 0,9146 \\
\hline$\frac{2}{D}$ & $\mathrm{HCM}$ & 16,9 & $14-20,3$ & 1,1 & 16,88 & $14,89-18,94$ & 1,11 & 0,125 & 0,5477 & - & - & - & - & - & - & - & - & - & - \\
\hline$\underset{>}{Z}$ & $\mathrm{CHCM}$ & 34,3 & $32-37$ & 1 & 36,68 & $37,1-40,05$ & 0,68 & 18,54 & 1 & - & - & - & - & - & 34,1 & $32,8-35,6$ & 0,7 & 1,34 & 0,9058 \\
\hline$\ddot{\nabla}$ & Leucocitos & 8,3 & $4,8-12$ & 1,7 & 8,86 & $5,29-13,75$ & 2,13 & $-2,04$ & 0,026 & 8,06 & - & 1,8 & 0,1647 & 0,5635 & 8,9 & - & 1,76 & 1,91 & 0,9719 \\
\hline$\overline{2}$ & Lym\# & 3,6 & $1,4-7,5$ & 1,2 & 2,9 & $1,1-4,19$ & 1,1 & 4,19 & 1 & 3,3 & - & 1,3 & 0,8 & 0,7881 & 4,19 & - & 1,4 & $-5,17$ & 1 \\
\hline$\frac{9}{8}$ & Mon\# & 0,4 & $0,2-0,7$ & 0,1 & 0,33 & $0,13-0,59$ & 0,11 & 4,64 & 1 & 0,25 & - & 0,16 & 3,39 & 0,9996 & 0,2 & - & 0,44 & 3,13 & 0,9991 \\
\hline 3 & PLT & 239 & $101-401$ & 68,2 & 114,55 & $46,37-193,87$ & 28,65 & 15,59 & 1 & - & - & - & - & - & 182 & $85-261$ & 43 & 5,86 & 1 \\
\hline
\end{tabular}

HB: Hemoglobina, HCT: Hematocrito, VCM: Volumen corpuscular medio, HCM: Hemoglobina corpuscular media, CHCM: Concentración de hemoglobina corpuscular media, PLT: Plaquetas. 


\subsection{Comparación de los resultados obteni- dos con referencias bibliográficas inter- nacionales}

Se Compararon los resultados con tres referencias internacionales: "Evaluación de hemogramas equinos usando ADVIA 120 comparado con el Contador por impedancia y conteo diferencial manual" (Giordano et al., 2008); "Valores hematológicos en caballos mestizos chilenos de silla" (Böhmwald et al., 1984) y "Valores hematológicos, bilirrubina y actividad enzimática en caballos peruanos de paso del valle de Lurín, Lima" (ver Figura 2 y Tabla 2) (Díaz et al., 2011).

\section{Discusión}

Al analizar la Tabla 2, comparando los resultados con Giordano et al. (2008) realizado en Italia, los valores de referencia del hematocrito y el volumen corpuscular medio encontrados en los caballos a más de 3000 m.s.n.m. presentaron diferencia significativa mayor, por lo que se podría atribuir a la altura. Al disminuir la presión atmosférica se disminuye también la presión parcial del oxígeno, produciendo hipoxia tisular la cual favorece la secreción de eritropoyetina, una glicoproteína que estimula la formación de eritrocitos (Hall, 2016).

Cuando se comparó con Böhmwald et al. (1984), realizado en Chile, se encontró que los valores de referencia de eritrocitos, hemoglobina y hematocrito encontrados en los caballos a más de 3000 m.s.n.m. presentaron diferencia significativa mayor, por lo que se podría asumir es debido a la altura para captar y movilizar el oxígeno y así suplir con los requerimientos tisulares normales (Hall, 2016).

Con el estudio de Díaz et al. (2011), realizado en Lima Perú, no se encontró diferencia significativa en los parámetros de eritrocitos, hematocrito, VCM, CHMC y HCM pero si en la concentración de hemoglobina, lo que podría ser atribuido a una característica racial de los caballos peruanos de paso, considerados como caballos de sangre caliente al tener origen de caballos españoles (Barré, 2016) al igual que los caballos criollos ecuatorianos.

Los resultados de los leucocitos totales y sus diferentes tipos en caballos criados o nacidos a más de 3000 m.s.n.m. fueron muy diversos comparados con las tres referencias internacionales. Kraft y Dürr, (2000), mencionan que cualquier influencia de la presión sanguínea, como su incremento por cualquier estímulo fisiológico, esfuerzo físico, patológico pueden provocar una alteración del recuento leucocitario y hemograma.

Los valores obtenidos de las plaquetas también presentaron resultados superiores comparados con los estudios de Giordano et al. (2008) y Díaz et al. (2011), lastimosamente no se disponían de valores en el estudio de Böhmwald et al. (1984). Es importante recalcar que los rangos normales en caballos son muy amplios (Cowell y Tyler, 2002; Weiss y Wardrop, 2010).

\section{Conclusiones}

Los valores de referencia de caballos nacidos o criados a más de 3000 m.s.n.m. en la serie roja presentaron diferencia significativa $\mathrm{P}>0.05 \%$ en los parámetros de glóbulos rojos, hemoglobina y hematocrito comparado con el estudio de Böhmwald et al. (1984); mientras que con el estudio de Díaz et al. (2011), solo presento diferencia significativa con la hemoglobina y en el estudio de Giordano et al. (2008), con el hematocrito. Esto puede deberse para facilitar el transporte de oxígeno en la sangre.

Con respecto la serie blanca, no hubo diferencia significativa con el conteo total de glóbulos blancos y granulocitos frente a los tres estudios, pero si una diferencia significativa en el recuento de monocitos, por lo que no se puede concluir que sea por efecto de la altura.

Los valores de referencia de caballos nacidos o criados a más de 3000 m.s.n.m. del contaje de plaquetas presentaron diferencia significativa contrastados con los estudios de Giordano et al. (2008) y Díaz et al. (2011), lastimosamente el estudio de Böhmwald et al. (1984), no disponía de información sobre recuento plaquetario. Es importante tener en cuenta que los valores plaquetarios pueden tener normalmente rangos muy amplios en caballos.

Finalmente, al comparar los valores del hemograma de caballos nacidos o criados a más de 3000 m.s.n.m. con el estudio de Díaz et al. (2011), en caballos peruanos de paso se observó que la mayoría de los parámetros no presentaron diferencias significativas. 


\section{Agradecimientos}

A las comunidades y propietarios de caballos que nos colaboraron para la obtención de muestras para la realización de este trabajo.

\section{Referencias}

Barranco, F., J. Blasco, A. Mérida, M. Muñoz, A. Jareño, J. Cozar, R. Guerrero, J. Gil, C. Martín y J. Rodríguez. 2002. Principios de urgencias, emergencias y cuidados críticos. SAMIUC [Versión de Uninet]. Andalucía. http://tratado.uninet.edu/

Barré, G. El caballo criollo. El caballo Peruano de Paso (Perú). 2016. http://www.justacriollo.com/pages_es/race screoles_es/Rcpaso_peruano_es.htm

Böhmwald, H., E. Wegmann y F. Witter. 1986. Valores hematológicos en caballos mestizos chilenos de silla. Universidad Austral de Chile. Valdivia. http://web.uchile.cl/vignette/monografiasv eterinaria/monografiasveterinaria.uchile.cl/

Cowell, R. y R. Tyler. 2002. Cytology and Hematology of the Horse. St.Louis: Mosby. páginas 191-207.

Cuenca, R. y J. Pastor. 2006. Utilidad del hemograma en la Clínica equina. Universidad Autónoma de Barcelona. España. 14:11-27.

Díaz, H., C. Gavidia, O. Li y A. Tío. 20011. Valores Hematológicos, bilirrubina y actividad enzimática sérica en caballos peruanos de paso del valle de Lurín. Rev. Inv Vet Perú. 22(3):213222.

Friedrichs, K., K. Harr, P. Kathy, K. Freeman, B. Szladovits, M. Raquel, R. Walton, K. Barnhart y J. Blanco. 2012. ASVCP Reference Interval Guidelines: Determination of de Novo Reference Intervals in Veterinary Species and other Related Topics. Veterinary Clinical Pathology. Madison. DOI:10.1111/vcp.12006
Geffré, A., D. Concordet, J-P. Braun y C. Trumel. 2011. Reference Value Advisor: a New Freeware Set of Macroinstructions to Calculate Reference Intervals with Microsoft Excel. Veterinary Clincal Pathology. Toulouse. 40(1):10712. DOI:10.1111/j.1939-165X.2011.00287.x, http:/ /onlinelibrary.wiley.com/doi/10.1111/ j.1939-165X.2011.00287.x/abstract

Giordano, A., G. Rossi, C. Pieralisi y S. Paltrinieri. 2008. Evaluation of Equine Hemograms Using the ADVIA 120 as Compared with an Impendance Counter and Manual Differential Count. Veterinary Clinical Pathology. Milano. 37(1):2130. DOI:10.1111/j.1939-165X.2008.00012.x, http:/ / onlinelibrary.wiley.com/doi/10.1111/ j.1939-165X.2008.00012.x/abstract;jsessionid= 4D046600560A5152AF9B91B1E00BD999.f02t0 1

Greene, H. M., E. A. Cogger, T. P. Anderson, C. C. Lewis, S. J. Wickler y A. 1999. Wyle. High-Altitude Effects on Respiratory Gases, Acid-Base Balance and Pulmonary Artery Pressures in Equids. Equine Veterinary Journal. Pomona. 30(S30):716. DOI:10.1111/j.2042-3306.1999.tb05192.x, https:/ / www.researchgate.net/publication/1 2656094_High_altitude_effects_on_respirator y_gases_acid-base_balance_and_pulmonary_ artery_pressures_in_equids

Hall, J.E. Guyton. 2016. Hall Text book of Medical Physiology. Elsevier. Philadelphia.

Kraft, W. y U. Dürr. 2000. Diagnóstico de laboratorio clínico en veterinaria. GRASS Ediciones. New York.

McGorum, C. B., Ed. Robinson, J. Schumacher y M. P. Dixon. 2007. Equine Respiratory Medicine and Surgery. Elsevier. Philadelphia. página 19.

Mindray BC-2800VET. Operators's Manual. Auto Hematology Analyzer. 2012. http:/ /www.mindray.com/es/products/45.h tml 